\title{
Performance Study of Tube Settlers Module
}

\author{
Amod Gurjar*, Mangesh Bhorkar**, Dr. A. G. Bhole***, Payal Baitule **** \\ *(M. Tech. Scholar Civil Engg. Dept., GHRCE, \\ **(Assistant Professor Civil Engg. Dept., GHRCE, \\ ***( Ex. Professor Civil Engg. Dept., VNIT, Nagpur) \\ ****(Lecturer Chemical Engg. Dept., KDK NPN Polytechnic,
}

\begin{abstract}
The water treatment plant plays an important role for supplying and purifying potable water. The present study is aimed to emphasize the performance of tube settler unit. A pilot scale model of sedimentation tank is prepared $\&$ installed for performance measurement. In conventional sedimentation tank the detention time required is 2-4 hrs. In view of increasing demand of water for society, a modification is used in water treatment plant i.e. high rate settling. The detention time is reduced up to $10-15 \mathrm{~min}$. which is very less as compare to conventional sedimentation tank. The average efficiency of turbidity removal is $70-80 \%$ in modified unit as compare to conventional tube settler unit.
\end{abstract}

Keywords - Clarification, Inclination, Sedimentation, Turbidity

\section{INTRODUCTION}

Water is the most important and more widespread chemical compound on the Globe. It is the only compound found on earth in the liquid state and the only compound found on Earth in all three states liquid, solid and gaseous at the temperatures normally found on the Earth. Water is a crucial factor of life on earth \& hence it is the only essential commodity for man's survival on the planet from among all components present in natural environment, is the most subjected to pollution.

Sedimentation is a physical treatment process that utilizes to separate suspended solids from water by gravity. This process is widely used in the first stage in surface water treatment to remove causing turbidity particles after coagulation and flocculation. Sedimentation is also used to recover water in filter backwash water recovery systems and to increase sludge solids concentration in sludge thickening. Pre sedimentation is also used in some cases to remove settleable solids such as gravel, grit, and sand from river water before it is pumped to the treatment plant. In water and wastewater treatment one of the oldest and most important problems of separation of suspended solids. The most widely used solids removal process is sedimentation, about one third of the total capital expense to its sedimentation tank of an entire water treatment plant. To reduce sedimentation costs several attempts have been made. Certain technique and advances are developed to reduce the cost and size of sedimentation units. One new advanced technique is high rate sedimentation, which reduces the detention time of settling device by reducing the distance of floc particles. In these devices normally parallel plates and tubes are placed horizontally or at an inclination at $60^{\circ}$ angles to the horizontal. (Bhole et. al.1996) To increase treatment capacity of clarifier tube settler systems are not expensive remedy for drinking water and wastewater plants, that with the use of tube settler decrease operating costs, improve effluent water quality and reduce new installation footprints. Constructed lightweight PVC tube settler is supported easily with minimum structures that effluent incorporates baffles and troughs. The settling capacity of rectangular sedimentation basins and or circular clarifiers increase with the use of tube settlers and parallel plates by reduce the vertical distance of floc particle are settle agglomerating to form particles of larger size. Tube settlers are use multiple tubular channels slope at a certain angle and related to each other, which combine and increased effective settling area. This provides for a settling depth of particle that is less than the settling depth and reducing settling times of a conventional clarifier unit.The use of laminar flow devices has been successful in reducing the settled water turbidity in some installations. These devices are often using to enhance the performance of existing but overloaded sedimentation basins. The use of shallow depth settling basins enables the detention time of settling process to be reduced to only a few minutes in contrast to conventional settling basin designs which use 1 to 4 hour detention. (Shirley E. clark et.al.2009)

\section{LITERATURE REVIEW}

Kshitija Balwan et al., 2016 Conducted a pilot scale model and installed at Ichalkaranji municipal water treatment plant the effect of length and inclination of tube settler the flocculated water 
was used the multiple tubular channels of tube settlers are used at an angle of about $45^{\circ}$ to $60^{\circ}$ and adjacent to that with the used of this the increased effective settling area the circular tubes were used with inclination of $45^{\circ}$ to $60^{\circ}$ of $45 \mathrm{~mm}$ diameter the length of tube varied as $60 \mathrm{~cm}, 50 \mathrm{~cm}, \& 40 \mathrm{~cm}$ the four pvc tubes of $4.5 \mathrm{~cm}$ diameter was connected to the bottom of base tank representing the tube settler.

A Faraji et al., 2013 Conducted a pilot scale model and installed at the exbatan wastewater treatment plant the polyvinyl chloride $(\mathrm{Pvc})$ material are used of $20 \mathrm{~cm}$ diameter at an angle 45 degrees related to horizontal the $60 \mathrm{~cm}$ length of tube in both the stages in conventional secondary clarifiers examine the possibility of applying one and two stage inclined tube settlers are used. The results shows that in 20 min detention time in the tubes the average removal of TSS, BOD, and COD in one stage tube settler pilot plants was $97.6 \%, 96.4 \%$, and $96.36 \%$ respectively while in other conventional secondary sedimentation basin was $98.2 \%, 99 \%$, and $98.6 \%$ respectively.

Shirley E. Clark et al., 2009 Reviewed the inclined plates/ tube settler in that overlapped plates are used for large settling area and also treated for multiple contaminants in that the flow operated in laminar flow conditions ,In this paper the work are carried out by investigating of inclined plate settlers to treatment the runoff in that Reynolds numbers ranged from 7.5to 50000 in that the used of settlers high removal of particle density achieved over the range of an Reynolds numbers in that the teree kleen device technology in inclined plate the results showed that the Reynolds number tested form the laboratory simulated storm water was capable of removing $>65 \%$ of solids are removed with the used of inclined plate settlers.

Cheng He et al., 2009 conducted vortex plate in an clarifiers instead of smooth lamellae in conventional the modeling and laboratory experiments was study by using computational fluid dynamics (CFD) in that crushed walnut shells \& glass beads were mimicked by suspended particles the vortex plates are forming an slots of 25 depth and 25 width by attached perpendicular ribs to the plate and the plate placing at an angle $60^{\circ}$ to the horizontal and plate parallel to an longitudinal clarifier axis a computational fluid dynamics was applied to clarifiers the result shows that the used of two vortex plate or an two smooth plates the vortex plates removing $8 \%$ more particles than the used of smooth plate with the used of vortex plate the particle removal will be more by larger inflow rates and for slower settling particles by up to $26 \%$.

Thomas ruby bentzen et al., 2009 In this paper studied the correlation between the associated pollutant concentrations and the settling velocity for better management practice in design, redesign ,or evaluate of an existing pond efficiency and retaining unwanted pollutants in this paper the polyaromatic hydrocarbon (PAH) concentration instead unknowns of two in settling velocity calculations the shapes \& density of single particle fraction the measurements shows that the highest concentrations is associated in cadmium ,chromium, zinc and nickel \& the lower concentration accumulated within the faster falling fraction in each pond the larger mass of metals and PAH can be found with a settling velocity of 5.5-2.5 at $19^{\circ} \mathrm{c}$ are found approximately.

A.G. Bhole et al., 1996 In this paper the suspended solids are removed. By sedimentation in a conventional clarifier. The depth of the conventional clarifier is about 2 to 3.5 meters and the detention time is in the range of 2 to 4 hours. Thus a lot of time is required in conventional clarifier for the settlement of flocks. The term high rate settlers refers to shallow gravitational settling devices with detention periods of the order of 10 to 20 minutes. The efficiency of high rate settlers is also more than that of conventional clarifier. The present study an attempt is an made to evaluate the relative performance of conventional and modified tube settlers for the four configurations of the tubes, namely circular, square, hexagonal and chevron the tubes were modified by reorienting them and by introducing partitions in the tubes. The tubes were fabricated such that the $\mathrm{c} / \mathrm{s}$, area of all the tubes was $25 \mathrm{~cm}^{2}$. Therefore the square tubes have $5 \mathrm{~cm}$ sides, circular tubes have $5.65 \mathrm{~cm}$ diameter, hexagonal tubes have $3.10 \mathrm{~cm}$ each side and chevron tubes have $4.20 \mathrm{~cm}$ each side are used. The percentage removal efficiency of the conventional tube settlers varied from 75 to $89 \%$ after six hours of observation depending on the turbidity and angle of inclination, whereas the percentage removal efficiency of the modified tube settlers varied from 82 to $97 \%$ for identical conditions.

\section{MATERIALS AND METHODS}

\subsection{Study area description}

The study is carried out to determine the performance of tube settler module. In that the tube settler unit is designed, fabricated and installed at water treatment plant to check the efficiency of unit and to compare conventional sedimentation (lamella plates) with tube settler unit.

\subsection{Set up information}

The set up is design and fabricated with MS sheet and installed at water treatment plant at Nagpur Region. The size and shape of tube for unit is square length of tube is kept as $1 \mathrm{~m}$ and is placed at an angle of $60^{\circ}$ inclinations with horizontal. The no. of tubes was 6 . The area for each tube is $25 \mathrm{~cm}^{2}$. Fig. 1 shows the cross section of tube shape and size is $5 \mathrm{~cm}$ breadth and $5 \mathrm{~cm}$ depth. The settler module \& Fig. 2 shows the actual pictorial view of setup. 


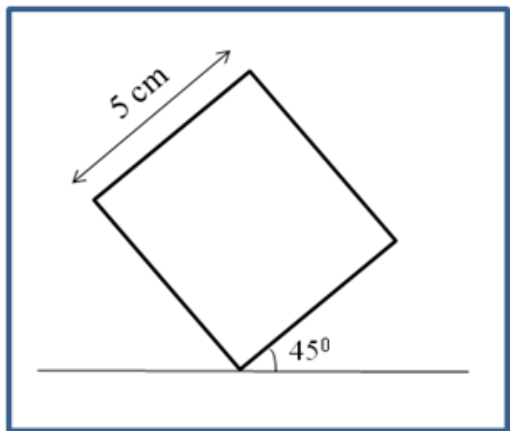

Fig. 1 Square Shape Tube Settler Module

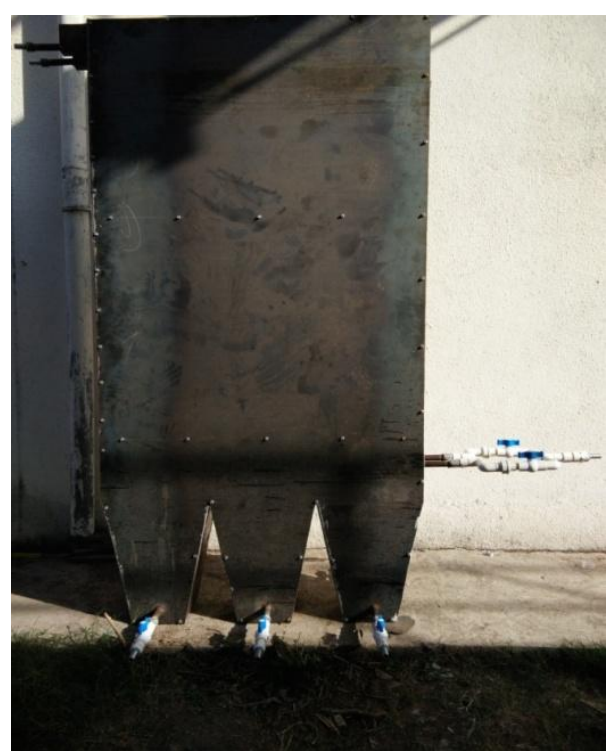

Fig. 2 Setup of Tube settler Module

\subsection{Experiment}

The pilot scale model of tube settler unit is installed in WTP. In the set up in inlet unit the flocculation tank water is carried out. The sludge outlet is provided on bottom of unit to remove the settled sludge. The samples were collected at the outlet of set up which is provided at top and checked the turbidity removal efficiency. The detention time was 15 minutes and rate of flow is $4 \mathrm{lpm}$.

\section{RESULTS AND DISCUSSION}

The results are carried out for tube settler module with the help of Nephlo Turbidity Meter. The turbidity removal efficiency is checked from October16 to February 17. The observations were recorded. The turbidity removal efficiency of unit is more as compare to conventional unit. The average efficiency of turbidity removal of unit is $70-80 \%$ more as compare to conventional unit. The modified tube settler unit has given quite good results. The results are shown in fig. 3 in graphical form.

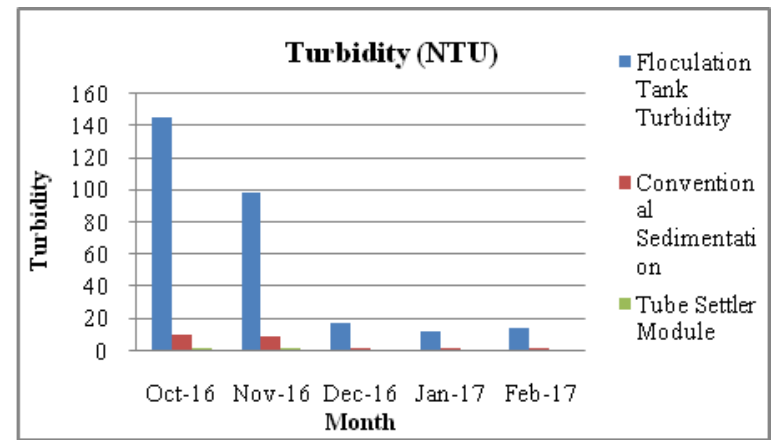

Fig. 3 Turbidity results 


\section{CONCLUSION}

From the above study, it is concluded that the turbidity removal efficiency in tube settler unit is high as compare to conventional sedimentation unit. The settling efficiency of particles is increased in tube settler module due to decrease in settling distance of particles. The average efficiency of turbidity removal is $70-80 \%$ in tube settler unit as compare to conventional unit.

\section{REFERENCES}

[1] A.G.Bhole et al., Journal of Indian water works association vol.28, No.1 (Jan.Mar 1996).

[2] A.Faraji et al., International journal of Civil Engineering, vol II, No 4, December 2013.

[3] Ahmed A. Fadel and Robert Baumann (1990) Tube settler modeling, Journal of Environmental Engineering, American Society for Civil Engineers,. Vol.116, No. 1, pp 107-124.

[4] American Water Works Association AWWA 1999 Water quality and treatment. A handbook of community water supplies, 5th edition, McGraw-Hill, New York.

[5] BRENTWOOD industries www.brentwood.com (August 2011) comparison between the tube settler and conventional settling.

[6] Cheng He et al., Journal of environmental engineering @ ASCE /August 2009.

[7] Clark SE, Roenning CD, Elligson JC, Mikula JB. Inclined plate settlers to treat storm-water solids, Journal of Environmental Engineering, ASCE, 2009, Vol. 135, pp.621-626.

[8] IS 10500: 1983, "Indian Standard for Drinking Water- Specification", Bureau of Indian Standards, New Delhi.

[9] Manual on Drinking Water Treatment and Supply (2000), Central Public Health and Environmental Engineering Organization. New Delhi.

[10] Shirley E.Clark et al., Journal of environmental engineering @ ASCE /August 2009.

[11] Thomas Ruby Bentzen et al., Journal of environmental engineering @ ASCE /November 2009.

[12] Kshitija Balwan et al., International journal of innovative research in advanced engineering (Issue 01, volume 3 January 2016). 